\title{
Participación social en salud en Pereira y Dosquebradas. Perspectivas de actores sociales e institucionales
}

\section{Social participation in health in Pereira and Dosquebradas. Perspectives of social and institutional actors}

\author{
Alexandra Agudelo-Ramírez (iD) ${ }^{1}$, Jorge Alexander Daza-Cardona (iD) ${ }^{2}$ \\ 1. Fundación Universitaria Autónoma de las Américas, sede Pereira. Correo: alexandra.agudelo@uam.edu.co - https://orcid.org/0000-0002-5858- \\ 8420 \\ 2. Fundación Universitaria Autónoma de las Américas, sede Pereira. Correo: jorge.daza@uam.edu.co - https://orcid.org/0000-0002-7343-7321
}

\begin{abstract}
Tipología: Artículo de investigación científica y tecnológica
Para citar este artículo: Agudelo-Ramírez A, Daza-Cardona JA. Participación social en salud en Pereira y Dosquebradas. Perspectivas de actores sociales e institucionales. Duazary. 2021 julio; 18(3): 243-258. Doi: https://doi.org/10.21676/2389783X.4232
\end{abstract}

Recibido en febrero 05 de 2021

Aceptado en mayo 04 de 2021

Publicado en línea en agosto 02 de 2021

\begin{abstract}
RESUMEN
Palabras

clave:

promoción de la

salud;

participación de

la comunidad;

participación

social;

corrupción;

empoderamient

o para la salud.

El objetivo de esta investigación fue comprender la participación social en salud en Pereira y Dosquebradas, dos municipios del departamento de Risaralda, Colombia, en el año 2018. Para esto se tomó como referente la perspectiva de líderes sociales, proveedores de salud, secretarías de salud y organismos de control. Se realizó una entrevista semiestructurada a 17 actores clave. Para la interpretación de datos se usó análisis temático y se hizo validación a partir de la triangulación de investigadores. Se encontró que los participantes reconocen la crisis del sistema de salud, las debilidades en cuanto a la intersectorialidad, la politiquería desde lo barrial hasta la administración municipal, la participación instrumentalizada y las diferencias entre las necesidades de las comunidades y la planeación de las instituciones. Se concluye que es necesario cualificar el trabajo intersectorial en el que se incluyan los mecanismos de participación existentes; sin embargo, estos no tendrán éxito sin la voluntad política de democratizar la participación y sin el empoderamiento ciudadano. En este sentido, es fundamental el fortalecimiento de las organizaciones sociales para consolidar movimientos por la salud que se articulen en torno a las necesidades regionales.
\end{abstract}

\section{ABSTRACT}

Keywords: Health Promotion; Community Participation; Social Participation; Corruption; Empowermen t.
This research aimed to understand Social Participation in Health in two municipalities of the department of Risaralda, Colombia, in 2018: Pereira and Dosquebradas. The focus was the perspective of social leaders, health providers, public servants, and control organizations. A semi-structured interview was conducted with 17 key actors. For data interpretation Thematic Analysis was used. For validation it was performed triangulation of the researchers. It was found that participants recognize the crisis of the health system, weaknesses in intersectoriality, politiking of different actors from neighborhood until local government, instrumentalized participation, and differences between the needs of the communities and the planning of the institutions. It is concluded that it is necessary to qualify intersectoral work that includes the existing participation mechanisms; however, these will not be successful without the political will to democratize participation and without citizen empowerment. In this sense, it is essential strengthening social organizations to consolidate health movements articulated around the regional needs. 


\section{INTRODUCCIÓN}

La participación social en salud (PSS) recobró interés a partir de los planteamientos de la Organización Mundial de la Salud (OMS) plasmados en la Declaración de Alma-Ata y la Carta de Ottawa. Estos documentos marcaron el inicio de los enfoques de atención primaria en salud (APS) y promoción de la salud, con los cuales se apunta a cambiar la concepción técnica y biomédica de las intervenciones institucionales de arriba hacia abajo hacia aquellas centradas en las comunidades a partir de la comprensión de la salud como una construcción social ${ }^{1}$.

En contraste, desde la década de los ochenta, América Latina fue el escenario de una serie de reformas constitucionales y de los sistemas sanitarios que tuvieron como consecuencia la mercantilización de la salud y el reforzamiento del modelo morbicéntrico de atención individual. Por ende, se adoptaron los preceptos neoliberales en detrimento de los lineamientos aportados por la $\mathrm{OMS}^{2}$.

A pesar de lo anterior, estas reformas tuvieron como consecuencia la ampliación de los mecanismos de participación social. En el caso colombiano, la Constitución de 1991 proclamó al país como un Estado social de derecho, lo que derivó en la expedición de leyes que reglamentaban mecanismos de participación de diversa índole ${ }^{3}$, entre los que se encuentran el cabildo abierto, las veedurías ciudadanas, los presupuestos participativos, y entre otros. Igualmente, en lo que respecta al Sistema General de Seguridad Social en Salud, existen normas que indican el derecho de los ciudadanos a hacer parte de la gestión, planificación y vigilancia del sistema. Así se crearon mecanismos específicos en salud como las asociaciones de usuarios y los comités de participación comunitaria en salud ${ }^{4}$. Además, estas leyes determinaron que los encargados de promover y facilitar la PSS serían los entes territoriales (secretarías de salud departamentales y municipales), las entidades promotoras de salud (EPS) y las instituciones prestadoras de salud (IPS) ${ }^{5,6}$.
Ahora bien, aunque los mecanismos de participación contemplados en las leyes son instrumentos para ejercer la PSS, es conveniente tener en cuenta que las organizaciones comunitarias y de la sociedad civil también son formas y estrategias de participación. Al respecto, cabe resaltar el papel de las Juntas de Acción Comunal y Local, los acueductos comunitarios, las asociaciones de discapacitados, los grupos juveniles, entre otros. Esto demuestra la importancia de reconocer las organizaciones de base para la generación de espacios democratizadores y de movilización social $^{7,8}$.

En este contexto, la PSS se entiende como la coproducción de bienestar y desarrollo social, la cual implica el reconocimiento de la acción política y social para generar condiciones de empoderamiento individual y colectivo ${ }^{9}$. Así, aunque incluye el derecho a participar en el sistema de salud, lo trasciende, toda vez que se materializa a partir de las prácticas sociales cotidianas de las comunidades y en las decisiones que se toman en la esfera pública ${ }^{9,10}$. De ahí que la participación sea un medio para buscar transformaciones en la distribución desigual del poder, con el fin de lograr equidad para la satisfacción de las necesidades humanas ${ }^{9}$.

El interés creciente en la PSS tiene que ver con la consolidación de sociedades democráticas, lo que está en consonancia con la búsqueda de equidad y justicia social, principios defendidos en el enfoque de los determinantes sociales de la salud (DSS). Este sostiene que la participación social no puede entenderse enmarcada exclusivamente en la atención sanitaria ni en el sector salud, dado que las inequidades dependen de múltiples causas ${ }^{1}$. Así, la PSS implica que las comunidades puedan aumentar su control en las iniciativas de promoción de la salud y en la modificación de los DSS a través del fortalecimiento de su acción, el involucramiento en la toma de decisiones y la construcción de capacidades $^{11,12}$.

Uno de los mecanismos de participación más abordados en las investigaciones es el de los consejos municipales de salud. Al respecto, se 
afirma que estos aún están lejos de alcanzar un grado deliberativo, ya que en la mayoría de los casos son cooptados por intereses burocráticos que dificultan la representación genuina de la ciudadanía. Además, prevalecen un lenguaje técnico y un enfoque medicalizado que otorga poder a los expertos en detrimento de otros grupos de interés ${ }^{13-}$ 17.

Asimismo, se ha encontrado que las personas se movilizan individualmente en busca de solución de eventos concretos, la participación institucional no funciona de acuerdo con sus propósitos, y existen manipulación y restricciones en el acceso a la información ${ }^{18,19}$. En un sentido similar, algunos trabajos revelan cómo la PSS aún es incipiente en cuanto a la planificación comunitaria y territorial, dado que persiste una concepción institucional y vertical que no contempla las acciones de base social ni las dinámicas particulares de los territorios $^{20-23}$. Sin embargo, algunas experiencias muestran el potencial de los mecanismos de participación formal, especialmente cuando se da el acompañamiento de las instituciones de salud y de los entes territoriales para promover la cooperación, la transparencia y la inclusión ${ }^{24,25}$.

De tal manera, es necesaria la comprensión de la PSS para fortalecer el cambio de enfoque en la formulación de políticas públicas y mejorar las intervenciones comunitarias y los servicios de salud, máxime cuando existe evidencia de que al promoverla se generan transformaciones sociales y reducción de inequidades ${ }^{26,27}$. Teniendo en cuenta este panorama, el objetivo de este artículo es comprender la PSS en dos municipios del departamento de Risaralda, Colombia, desde la perspectiva de líderes sociales, proveedores de salud, secretarías de salud y organismos de control.

\section{MATERIALES Y MÉTODOS}

El presente estudio fue cualitativo con diseño hermenéutico, y este busca incrementar el entendimiento de las culturas, los grupos sociales y los individuos tratando la realidad social como un "texto" susceptible de interpretación a partir de la comprensión del "mundo" que rodea su producción. En otras palabras, se parte de la premisa de que el ser humano realiza actos simbólicos que cobran sentido en contextos sociales e históricos ${ }^{28}$. Así, la PSS se comprendió a la luz de la perspectiva de múltiples actores, cuyos discursos se enmarcan en un conjunto de relaciones políticas, económicas y sociales a nivel continental, nacional y local. Por otro lado, el trabajo de campo fue realizado en el año 2018.

\section{Participantes}

La investigación tuvo como alcance comprender la PSS en dos municipios del departamento de Risaralda, Colombia: Pereira y Dosquebradas. Estos municipios mantienen un intenso intercambio dada su cercanía geográfica, lo que genera interdependencia en múltiples esferas. Se buscaron actores clave que por su rol institucional o comunitario pudieran dar cuenta de la dinámica de la PSS. Con respecto a los actores institucionales, se incluyeron funcionarios de las secretarías de salud, hospitales y personerías. En cuanto a los actores comunitarios, se contactaron líderes pertenecientes a dos de los sectores más grandes de ambos municipios: El Dorado en Pereira y Frailes en Dosquebradas. La elección de estos sectores respondió al interés de generar una interpretación que tuviera en cuenta simultáneamente las dinámicas municipales y barriales, generando un movimiento dialéctico entre lo macro y lo micro. En total, participaron 17 personas: nueve representantes institucionales y ocho comunitarios.

\section{Procedimientos}

La técnica de recolección de información fue la entrevista semiestructurada. Las preguntas fueron diseñadas por los investigadores en congruencia con otras investigaciones latinoamericanas sobre PSS. Sin embargo, se dio prioridad al discurso de los participantes, quienes tenían libertad para introducir cuestiones que estuvieran por fuera del guion. Los interrogantes giraron en torno a los siguientes temas: conocimiento de las comunidades sobre el sistema de salud, particularidades locales de la participación, organización comunitaria, actores protagónicos y excluidos, motivaciones para participar, uso de mecanismos formales e informales, barreras y facilitadores de la 
participación, intersectorialidad, capacidad instalada y experiencias exitosas.

\section{Análisis de datos}

Las entrevistas fueron grabadas y transcritas. Se utilizó análisis temático ${ }^{29}$ a partir de una selección de unidades de sentido, las cuales se codificaron y organizaron en una matriz de saturación. Los códigos fueron clasificados por temas, proceso en el que se hizo énfasis en la emergencia de categorías. Posteriormente, en un movimiento circular entre lo general y lo particular, las categorías fueron puestas a prueba frente a las unidades de sentido hasta que se construyó una narrativa que diera verosimilitud a la perspectiva de los actores.

Para la validación de los resultados se realizaron dos procedimientos: triangulación desde la perspectiva de los investigadores y devolución sistemática de los resultados con los participantes ${ }^{30}$. La triangulación de los investigadores contó con un equipo de cuatro personas: los dos autores del presente texto, una experta en el funcionamiento del sistema de salud y una estudiante de medicina de último año con formación en salud comunitaria; cada miembro del equipo contribuyó al análisis de las entrevistas e hizo una retroalimentación al trabajo realizado por los otros miembros. Para la devolución sistemática de los resultados, se organizó un evento al que se invitó a los líderes comunitarios y a los representantes de las instituciones participantes. En este se mostraron las categorías y los códigos que las ejemplificaban.

\section{Declaración sobre aspectos éticos}

Se siguieron los principios éticos de la Declaración de Helsinki y la Resolución 8430 de 1993 del Ministerio de Salud de Colombia, según la cual se clasifica como un estudio de riesgo mínimo. Cada participante recibió explicaciones sobre la investigación y firmó el consentimiento informado. Asimismo, se contó con el aval del Comité de Ética en Investigación de la Fundación Universitaria Autónoma de las Américas.

\section{RESULTADOS}

De las personas entrevistadas, una era funcionario público de la Secretaría de Salud departamental y ocho eran funcionarios públicos de Dosquebradas y Pereira. De estos, en cada municipio participó un representante de la Secretaría de Salud, uno de la Personería y dos del hospital. Los funcionarios fueron tres hombres con edad promedio de 50 años y seis mujeres con edad promedio de 40 años, todos profesionales. Asimismo, se entrevistaron cuatro líderes comunitarios de cada municipio, cuatro hombres y cuatro mujeres con edad promedio de 50 años y 43 años respectivamente, solo tres de ellos con nivel de educación superior. En la figura 1 se muestran las categorías y subcategorías según el análisis inductivo.

\section{Crisis del sistema de salud}

Todos los actores plantearon que el sistema de salud está en crisis porque hay dilaciones excesivas en la atención y se niegan medicamentos, procedimientos y cirugías que las personas requieren. Adicionalmente, percibieron que el talento humano es insuficiente y tiene sobrecarga asistencial, por lo cual no tiene tiempo de realizar acciones de promoción de la salud. A esto se suman la escasa vigilancia de los entes territoriales y la poca maniobrabilidad de los organismos de control dado que las EPS son de carácter privado.

Frente a esta situación, varios de los actores refirieron que la exigencia de la atención se trasladó a la rama judicial. Así, ante la falta de respuesta institucional los ciudadanos acuden a las vías judiciales, aunque estas también están colapsadas, como lo afirmó un representante de la Personería: "No se están cumpliendo las tutelas, entonces pasamos a los incidentes de desacato; al no cumplirlos nos toca presentar denuncias penales, pero nada de esto surte efecto positivo". Al respecto, un líder comunitario comentó que un abogado de la Superintendencia de Salud le dijo: "Le voy a dar un consejo: no se enferme". 


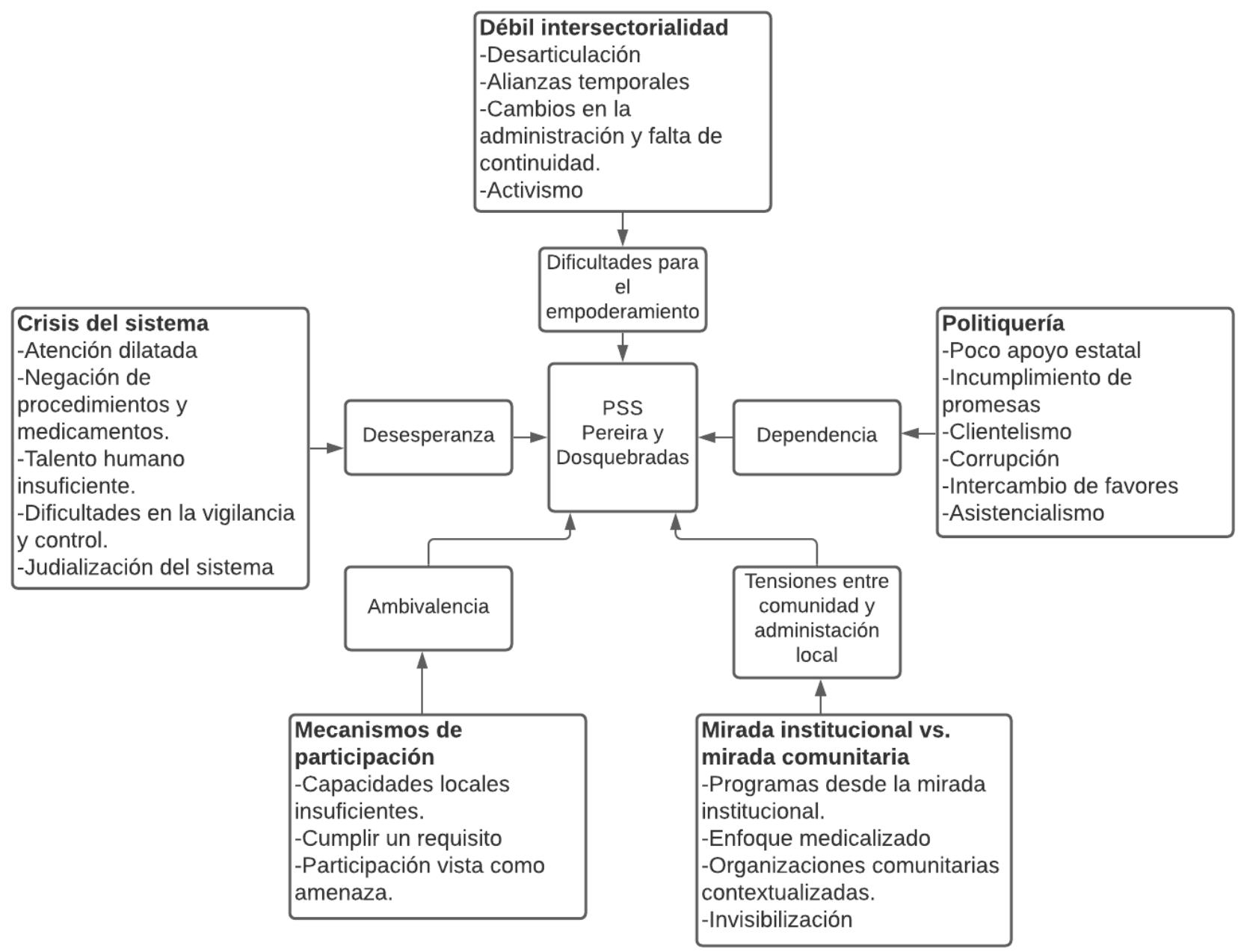

Figura 1. Diagrama de categorías y subcategorías a partir del análisis.

Por lo anterior, algunos funcionarios ven poco útil la participación de los líderes comunitarios, pues consideran que el sistema no responde a la organización social, sino a conocimientos en derecho. Por su parte, ciertos líderes manifestaron que se sienten "atropellados" por el sistema y que necesitan conocer su funcionamiento para defender sus derechos. Sin embargo, otros han perdido las esperanzas en que los mecanismos de participación cambien su situación debido a las experiencias negativas de personas cercanas que no lograron acceso a la atención.

\section{Débil intersectorialidad}

Una debilidad evidenciada fue la falta de intersectorialidad. Las instituciones no están articuladas con las organizaciones comunitarias, así como tampoco hay trabajo coordinado interinstitucional, intrainstitucional, $\mathrm{ni}$ en los diferentes niveles territoriales (local-regionalnacional). Sin embargo, existen acciones que se realizan conjuntamente, pero son temporales.

Asimismo, se percibe desarticulación entre las acciones de promoción y prevención del régimen contributivo y las del subsidiado, lo que genera que se deterioren los procesos con las comunidades. Esto se da principalmente cuando un usuario cambia del régimen subsidiado al contributivo, pues queda desvinculado de las acciones comunitarias agenciadas por los puestos de salud que solo atienden al régimen subsidiado.

Aunado a lo anterior, la desarticulación se agrava con los cambios de las administraciones municipales 
y departamentales debido a que la llegada de otro partido político al poder implica que se dejen de lado los programas que estaban en marcha. Igualmente, las formas de contratación afectan el trabajo con las comunidades dado que los contratistas no están empleados el año completo, lo que conlleva a que los programas queden sin respaldo institucional por varios meses y que las acciones se conviertan en activismo para entregar resultados en poco tiempo.

También sucede que se duplican los esfuerzos y la inversión de recursos porque cada contratista busca sus grupos de interés y no se trabaja en equipo, a pesar de ser parte de una misma institución. Un ejemplo de esto es lo mencionado por un representante de una secretaría de salud: "cuando te das cuenta de que tienes un Plan de Intervenciones Colectivas y tienes una figura de cultura y deportes de un municipio y ambos están haciendo lo mismo, tú dices 'Aquí falta articulación'”.

Por lo tanto, la falta de coordinación de las acciones institucionales y la fragmentación del sistema de salud son barreras que minan el fortalecimiento de redes comunitarias, cortan la financiación de los programas, reducen el impacto de las intervenciones y dificultan el empoderamiento de las comunidades debido a la carencia de procesos a largo plazo.

\section{Politiquería en la PSS}

Los líderes fueron enfáticos al plantear que, a pesar de sus gestiones, la PSS tiene poco apoyo estatal. Así, la falta de financiación ocasiona que la mayoría de sus propuestas desfallezcan y los gestores queden agotados por intentar sostenerlas. Esta situación se agrava con el incumplimiento de las promesas hechas por funcionarios y políticos, lo cual genera desconfianza frente a la función pública y deja "heridas muy grandes y difíciles de cerrar". Finalmente, al no ver otras posibilidades, muchos líderes optan por recurrir a "los amigos políticos" para lograr sus propósitos, dinámica que alimenta el tráfico de influencias y el clientelismo.
La politiquería incluye la vinculación de las comunidades en campañas electorales a la espera de recibir beneficios, en un círculo vicioso de clientelismo y corrupción, tal como lo manifestó un líder comunitario: "La gente sídemanda, pero es que la manera de demandar es trabajar con el político para que luego le pague el favor a la comunidad haciéndole permisos de obra". Esto refleja un tipo de participación manipulada que se basa en el asistencialismo y el intercambio de favores. Al respecto, un líder comunitario expresó lo siguiente: "Estamos en un Estado asistencialista, es una estructura que le interesa que tenga pobres, necesitados y que se vuelvan limosneros. Entonces lo que nosotros queremos es cambiar el chip y llevar a la gente a que construya ciudadanía, a que construya comunidad, y a que no dependan de lo que otro les da, sino que ellos generen".

Además, para muchos de los entrevistados la participación en la formulación de políticas públicas está viciada, pues lo que interesa a los políticos es "la foto y la firma de asistencia". La expresión de un líder comunitario es contundente en este sentido: "El Plan Municipal de Desarrollo lo hacen al principio de la administración pa' tomar una foto y decir que el plan de gobierno fue construido de manera participativa, pero ese man ya sabe a qué se va a subir allá".

Por otra parte, la politiquería ha permeado las prácticas de algunos líderes cuando ejercen el control social. Por ejemplo, una funcionaria mencionó que los veedores "si encuentran un hallazgo fiscal, le piden un contrato al servidor público para no denunciarlo", hecho que da cuenta de la forma en que la cultura de la corrupción permea gran parte de la vida cotidiana. Otra de las narrativas da cuenta de ello: "Un fenómeno que yo analizo con los líderes es que la casa del líder tiene buena presentación, está bien dotada por dentro; ese líder está beneficiándose, pero no le está ayudando a la comunidad".

En Pereira la percepción de la politiquería en la participación es menor que en Dosquebradas. Por un lado, porque se ha naturalizado el hecho de que beneficios, obras y proyectos se consiguen a través de amigos políticos, de manera que muchos no lo 
interpretan como un aspecto negativo. Por otro lado, porque hay algunas experiencias, como la del comité de salud de El Dorado, blindadas a intereses electorales que han traído resultados positivos para todas las partes. Aunado a esto, en la capital queda la sensación de haber alcanzado resultados con las gestiones de los líderes ante las instituciones, asunto que se ve reflejado en la mejora de casetas comunitarias y la dotación del puesto de salud a través de presupuestos participativos. Mientras tanto, en Dosquebradas son pocas las experiencias positivas que disminuyen esta percepción.

\section{Uso de los mecanismos de participación}

Los funcionarios del sector salud reconocen la existencia de leyes para promover la participación. Sin embargo, algunos expresaron que la dificultad en su aplicación radica en que a nivel local y regional los encargados no están capacitados o no tienen voluntad política. Un funcionario hizo una autocrítica al respecto: "Siempre hemos fracasado en implementar estos grupos, por decir algo, las veedurías, IOS COPACO [Comités de Participación Comunitaria] y vemos que la manera de llegar a conformarlos no ha sido la más adecuada porque han estado temporalmente y no hay un proceso". De tal forma, hay funcionarios que conciben los COPACO, las rendiciones de cuentas y las asociaciones de usuarios como mecanismos que tienen que funcionar por dar cumplimiento a la ley, pero no como un ejercicio de derechos.

En cuanto a las asociaciones de usuarios de los hospitales públicos de ambos municipios, cabe destacar que ofrecen capacitaciones a sus miembros, promueven la autogestión de los líderes y generan espacios para recibir quejas de los usuarios frente a los servicios de salud. Sin embargo, en ocasiones los altos cargos de las instituciones perciben este mecanismo como una amenaza cuando los ciudadanos ejercen una participación crítica y realizan veedurías, de manera que se crean las asociaciones, pero no se fortalecen porque se piensa que "se pueden volver contra el hospital". Así, los profesionales encargados de promover y acompañar estos escenarios de PSS se sienten "entre la espada y la pared" porque deben cumplir tanto a sus empleadores como a la comunidad.

\section{Mirada institucional versus mirada comunitaria}

Varios participantes explicitaron que los programas y proyectos para promover la participación están pensados desde intereses y lógicas institucionales, y pocas veces tienen en cuenta las necesidades $y$ voces de las comunidades. Hay una debilidad en la conceptualización de la PSS desde la que parten las intervenciones sociales, así como en la programación del tiempo para los procesos de planeación y evaluación, dado que el énfasis está en la ejecución. Además, no se vincula a las comunidades en dichas etapas, en tanto son percibidas como receptoras de intervenciones.

Un aspecto en el que se ha avanzado es contratar equipos interdisciplinarios para realizar los planes de intervención colectiva. No obstante, los funcionarios reconocen que se enfocan en visitas puerta a puerta, ampliación de cobertura, registro y carnetización, mas no en promover propiamente la PSS. Además, en las intervenciones se continúa replicando un enfoque medicalizado y asistencialista con vacíos pedagógicos y comunicacionales. A pesar de ello, se encontraron dos experiencias exitosas de trabajo comunitario: un programa para la disminución del dengue y del chikunguña, y otro denominado Cocinas sin Humo, ambos con enfoque en ambientes saludables. Los funcionarios reconocen que el éxito de estos radica en que los servidores públicos "se ponen las botas" y trabajan mancomunadamente con la comunidad.

Otro aspecto mencionado por una parte de los servidores públicos fue la distancia en la comprensión de los lenguajes y la cultura de las comunidades, pues las intervenciones se realizan con términos técnicos, sin adaptarse a los saberes populares, ni a los tiempos comunitarios. Una anécdota compartida por un funcionario ejemplifica varios de esos asuntos: "Estábamos trabajando estrategias de prevención de embarazo en adolescentes y habíamos gastado mucha plata en eso, y se nos ocurrió invitar a cuatro adolescentes al salón donde estábamos supuestamente los sabios. Nos dieron una bofetada en la cara, pues nos dijeron 'Los escuchamos a ustedes todo el día y les 
agradecemos mucho que nos hayan invitado, pero lo que ustedes hicieron no es lo que nos gusta y por eso es que no hacemos lo que ustedes pretenden'. Se cayó todo porque no los tuvimos en cuenta, ni les dábamos la palabra".

En contraste, las organizaciones comunitarias que han generado intervenciones teniendo en cuenta los lenguajes y las necesidades de las comunidades han asegurado su aceptabilidad y pertinencia, como el caso de Sexo Abierto y Positivo, estrategia creada por jóvenes profesionales para otros jóvenes del sector de Frailes. Adicionalmente, la participación de este grupo etario resulta esquiva para los representantes institucionales, mientras que en las comunidades ellos son agentes activos. De hecho, un logro percibido es que en los procesos participativos se han formado nuevos líderes, algunos desde su niñez o adolescencia.

Frente a la concepción de la PSS, muchos funcionarios piensan que la promueven cuando aseguran la asistencia de la comunidad a una reunión y "ven el salón lleno"; otros, cuando se realizan talleres y reparten volantes, o cuando los líderes replican la información del puesto de salud. Estas concepciones reflejan una visión unilateral de producción de conocimiento, en la que el papel de los líderes es de reproductores, y el de las comunidades, de receptoras pasivas.

En este orden de ideas, ciertas posiciones institucionales asumen que la gente no está interesada en la salud pública ni quiere participar. Sin embargo, se invisibiliza la gestión de las comunidades en situaciones que tienen relación directa con la salud. Ejemplo de ello es la preocupación por el sostenimiento de los escenarios deportivos, el cuidado de los guaduales, la calidad del agua del acueducto comunitario, la contaminación de las quebradas, entre otros. Esto devela una concepción de la salud de ciertos funcionarios reducida a la morbilidad y su atención médica.

En contraste con la mirada institucional, los líderes entrevistados muestran una visión de la PSS mucho más amplia, relacionada con la promoción de la salud y el bienestar, ya que al abordar este tema inmediatamente referencian las jornadas de rumboterapia, comedores para adultos mayores, gimnasios públicos, mantenimiento de escenarios de uso colectivo, jornadas de adopción y esterilización de animales de compañía, caminatas ecológicas, biblioteca comunitaria, proyectos de educación sexual, acueducto comunitario, entre otras. Casi todas estas acciones se realizan desde las organizaciones de base comunitaria, y solo algunas son apoyadas por instancias institucionales. Respecto a las organizaciones, los actores mencionaron grupos deportivos, culturales, religiosos y musicales, asociaciones de jóvenes, banda marcial, movimiento LGBTI, club de lectura, asociación de discapacitados, juntas de acción comunal y asociación de madres comunitarias.

\section{DISCUSIÓN}

La OMS plantea que para fortalecer la PSS se deben trabajar por lo menos cuatro elementos: institucionalización de los mecanismos de participación, provisión de recursos, fortalecimiento de la capacidad de acción y revisión de prácticas de los funcionarios públicos ${ }^{31}$. En cuanto al primer elemento, es claro que en Colombia se han creado diversos mecanismos de participación, pero esto no es suficiente, como se mostró en esta investigación, pues los actores sociales se enfrentan a barreras burocráticas, falta de conocimientos técnicos, manipulación por las instituciones y estigmatización. En cuanto al segundo elemento, una de las barreras más mencionadas por los líderes comunitarios en este estudio fue la falta de recursos para sostener la participación a largo plazo.

Con respecto al tercer elemento, este trabajo evidencia la necesidad de superar inequidades en el acceso y la comprensión de la información, tanto del funcionamiento de los mecanismos de participación como del sistema de salud, de manera que los ciudadanos incrementen su empoderamiento $y$ puedan abogar por sus intereses en relaciones más horizontales con los agentes institucionales. Finalmente, en el cuarto elemento, cabe anotar que la trayectoria de relaciones comunidad-Estado se ha vivido de manera diferente en los dos municipios analizados $y$, por ende, las concepciones $y$ estrategias de participación se presentan de formas 
distintas. Sin embargo, ambos contextos están permeados por la historia de clientelismo que ha marcado a la participación en Colombia, como consecuencia de un sistema político cerrado y un modelo de sociedad excluyente ${ }^{32}$.

Habría que decir también que las voces de los entrevistados muestran que el marco legal y los mecanismos de participación formal son insuficientes para garantizar la PSS. El sistema está burocratizado, las tutelas y los desacatos son ignorados, e incluso los entes de control no siempre logran la defensa de los derechos ciudadanos. Esto se debe a que las lógicas del mercado han socavado la posibilidad de desarrollar la salud como un bien público. Así, se entiende que por sí mismas las normas no fomentan la participación si se sigue pensando la salud como un negocio centrado en la atención individual ${ }^{33}$.

Por otro lado, se debe entender que la PSS es desigual en los territorios, lo que se debe en parte a las medidas concretas que se toman en cada nivel político-administrativo, como también a elementos histórico-sociales de las comunidades y de sus relaciones con los gobiernos e instituciones locales $^{10}$. Este aspecto se evidenció en las diferentes acciones intersectoriales en el contexto barrial y local, así como en las percepciones desiguales sobre la politiquería dados los resultados de la gestión de los líderes comunitarios en ambos municipios. De este modo, a partir de la comprensión de manifestaciones disímiles de PSS en cada contexto sociocultural, se podrían formular políticas públicas y desarrollar intervenciones participativas en salud que superen la visión deficitaria desde la que se invisibilizan las acciones, las gestiones y los intereses de los sujetos para mejorar su salud. Esto último se plantea teniendo en cuenta que los líderes comunitarios mostraron una visión mucho más amplia de participación en salud que los agentes institucionales.

En contraste con otras investigaciones que se han centrado en los mecanismos de participación formal ${ }^{14-19,24,25}$, el enfoque de la presente investigación permitió interpretar la riqueza de la acción política y social de las organizaciones de base en los barrios, de la participación de los diversos actores en los escenarios de la vida cotidiana donde se construye el bienestar, y de la complementariedad de mecanismos formales y no formales. Entretanto, se necesitarán futuras indagaciones respecto a la reciente ley promulgada sobre PSS en el sistema de salud ${ }^{34}$, en términos de su impacto en la democratización de los espacios de deliberación y en el empoderamiento de la ciudadanía.

Finalmente, uno de los aspectos clave por fortalecer es el trabajo local intersectorial, que puede desarrollarse a través de mecanismos de participación ya existentes, como los Consejos Territoriales de Seguridad Salud y los COPACO. Al respecto, en algunos países los proyectos liderados desde el ámbito local institucional han sido importantes para incrementar el control de la población sobre sus determinantes de salud ${ }^{35,36}$. Sin embargo, se requiere voluntad política para democratizar estos escenarios de participación, así como ciudadanos empoderados que no caigan en trampas de manipulación. En este escenario, se plantea que el camino es el fortalecimiento de la organización social y la formación de ciudadanía crítica a través de la educación popular. De igual forma, esto debe articularse a los movimientos sociales en salud ${ }^{37}$ para descentralizar su acción de las principales ciudades del país y de los intelectuales de la salud, y generar alianzas con otros sectores, movimientos y organizaciones comunitarias.

\section{DECLARACIÓN SOBRE CONFLICTOS DE INTERESES}

Los autores declaran que no existe conflicto de intereses.

\section{CONTRIBUCIÓN DE LOS AUTORES}

Primer autor: diseño de proyecto, trabajo de campo, análisis de datos, redacción y revisión crítica del artículo.

Segundo autor: apoyo en trabajo de campo, revisión de codificaciones, redacción y revisión crítica del artículo. 


\section{REFERENCIAS BIBLIOGRÁFICAS}

1. Rasanathan K, Villar Montesinos E, Matheson D, Etienne C, Evans T. Primary health care and the social determinants of health: essential and complementary approaches for reducing inequities in health. J Epidemiol Community Health. 2011; 65(8): 656-60. Doi: http://dx.doi.org/10.1136/jech.2009.093914

2. Carmona-Meza Z, Parra-Padilla D. Determinantes sociales de la salud: un análisis desde el contexto colombiano. Salud Uninorte [revista en la Internet]. 2015; 31(3): 608-20. Disponible en: https://www.redalyc.org/pdf/817/81745378017.pd f

3. Colombia. Congreso de la República. Ley 1757 de 2015 por la cual se dictan disposiciones en materia de promoción y protección del derecho a la participación democrática. Diario Oficial, 49565 (Jul. 6 de 2015).

4. Colombia. Ministerio de Salud. Decreto 1757 de 1994 por el cual se organizan y se establecen las modalidades y formas de participación social en la prestación de servicios de salud. Diario Oficial, 41477 (Ago. 05 1994).

5. Colombia. Congreso de la República. Ley 1438 de 2011 por medio de la cual se reforma el Sistema General de Seguridad Social en Salud y se dictan otras disposiciones. Diario Oficial, 47957 (Ene. 19 2011).

6. Colombia. Congreso de la República. Ley 1751 de 2015 por la cual se regula el derecho fundamental a la salud y se dictan otras disposiciones. Diario Oficial, 49427 (Feb. 16 2015).

7. López-Bolaños L, Campos-Rivera M, VillanuevaBorbolla, MÁ. Compromiso y participación comunitaria en salud: aprendizajes desde la sistematización de experiencias sociales. Salud Publica Mex. 2018; 60(2): 192-201. Doi: https://doi.org/10.21149/8460

8. Villanueva-Borbolla MÁ, Cervantes-Ortega E, Campos-Rivera M. Análisis de la capacidad de respuesta comunitaria para desarrollar iniciativas de promoción de la salud. Glob Health Promot. 2020; 27(4): $\quad$ 187-97. Doi: https://doi.org/10.1177/1757975919893982

9. Carmona-Moreno LD. Concepción de la participación social en salud: propuesta de resignificación. Revista Ciencias de la Salud. 2017; 15(3): $\quad$ 441-54. Doi: http://dx.doi.org/10.12804/revistas.urosario.edu.c o/revsalud/a.6127

10. Barbieri N, Gallego R, Morales E, MuñozMendoza C, Terés BQ. Facilitators and challenges of community action for health. Comparative analysis of four case studies in neighbourhoods of Barcelona. Policy Studies. 2021; 42(3): 1-18. Doi: https://doi.org/10.1080/01442872.2019.1645323

11. Jackson SF, Birn AE, Fawcett SB, Poland B, Schultz JA. Synergy for health equity: integrating health promotion and social determinants of health approaches in and beyond the Americas. Rev Panam Salud Pública [revista en la Internet]. 2013; 34(6): 473-80. Disponible en: https://www.scielosp.org/article/rpsp/2013.v34n6/ 473-480/en/

12. Goodman RM, Speers MA, McLeroy K, Fawcett S, Kegler $\mathrm{M}$, Parker $\mathrm{E}$, et al. Identifying and Defining the Dimensions of Community Capacity to Provide a Basis for Measurement. Health Educ Behav. 1998; 25(3): 258-78. Doi: 10.1177/109019819802500303

13. Santana F, Van Stralen CJ, Antunes PH. Participação social e saúde no Brasil: revisão sistemática sobre o tema. Cien Saude Colet. 2014; 19(2): 487-98. Doi: https://doi.org/10.1590/141381232014192.10542012

14. Souza CMN, Heller L. Efetividade deliberativa em conselhos municipais de saneamento e de saúde: um estudo em Belo Horizonte-MG e em Belém-PA. Cien Saude Colet. 2019; 24(11): 4325-34. Doi: https://doi.org/10.1590/1413-

812320182411.03632018

15. Sáinz-Ruiz PA, Mínguez-Arias J, Martínez-Riera JR. Los consejos de salud como instrumento de 
participación comunitaria en La Rioja. Gac Sanit. 2019; 33(2): 134-40. Doi: https://doi.org/10.1016/j.gaceta.2017.09.012

16. Ruano AL, Sebastián MS, Hurtig AK. The process of social participation in primary health care: the case of Palencia, Guatemala. Health Expect. 2011; 17(1): 93-103. Doi: https://doi.org/10.1111/j.13697625.2011.00731.x

17. Caballero EG, Rodríguez JC, Peralta N, Flores L. Proyecto y situación de la participación social en el sistema de salud paraguayo. Novapolis [revista en la Internet]. 2017; (11): 115-35. Disponible en: http://pyglobal.com/ojs/index.php/novapolis/articl e/view/80

18. Puerta-Silva C, Agudelo-Calle A. Modalidades de interacción en el régimen subsidiado de salud en Medellín: participación y estrategias sociales. Hacia la Promoción de la Salud [revista en la Internet]. 2012; 17(1): 13-28. Disponible en: https://www.redalyc.org/pdf/3091/309124894008. pdf

19. Castañeda GI, Delgado ME. Barreras, oportunidades y tácticas para participar en salud según Asociaciones de Usuarios del Valle del Cauca, Colombia. Hacia la Promoción de la Salud. 2015; 20(2): $\quad 59-76 . \quad$ Doi: https://doi.org/10.17151/hpsal.2015.20.2.6

20. Carrillo-Franco J, López L. Participación social en salud en la atención primaria en Bogotá: voces de mujeres. Revista Gerencia y Políticas de Salud. 2014; 13(26): 144-57. Doi: https://doi.org/10.11144/Javeriana.RGYPS1326.PSSa

21. Merino CE, Torres MC. Participación en salud y desarrollo territorial: experiencia sanitaria en una comuna del sur de Chile. Ciencia y Enfermería. 2015; 21(1): 115-25. Doi: http://dx.doi.org/10.4067/S071795532015000100011

22. Rojas DE, Vejar LI, Rioseco RC, Carrasco PM, Espinoza PG. Participación social en la atención primaria en salud: tensiones y contradicciones. Aten
Primaria. 2020; 52(10): 690-6. Doi: https://doi.org/10.1016/j.aprim.2020.02.013

23. Giménez E, Rodríguez JC, Peralta N. Espacios de decisión en la descentralización de salud del Paraguay. Mem Inst Investig Cienc Salud [revista en la Internet]. 2016; 14(3): 44-51. Disponible en: https://pesquisa.bvsalud.org/portal/resource/pt/bi blio-869105

24. Nigenda-López G, Juárez-Ramírez C, Ruiz-Larios J, Herrera CM. Participación social y calidad en los servicios de salud: la experiencia del aval ciudadano en México. Rev Saúde Pública [revista en la Internet]. 2013; 47(1): 44-51. Disponible en: https://www.scielosp.org/article/rsp/2013.v47n1/4 4-51/

25. Jeri-De-Pinho, M. Juntas de Usuarios de los Servicios de Salud en el Perú: conocimientos, percepciones y prácticas sobre la protección de sus derechos. Anales de la Facultad de Medicina. 2018; 79(1): 17-21. Doi: http://dx.doi.org/10.15381/anales.v79i1.14587

26. Bath J, Wakerman J. Impact of community participation in primary health care: what is the evidence? Aust J Prim Health. 2015; 21(1): 2-8. Doi: https://doi.org/10.1071/PY12164

27. Fiorati RC, Arcêncio RA, Segura J, Ramasco M, Serrano P. Intersectorality and social participation as coping policies for health inequities-worldwide. Gac Sanit. 2018; 32(3): 304-14. Doi: https://doi.org/10.1016/j.gaceta.2017.07.009

28. Katayama RJ. Introducción a la investigación cualitativa: fundamentos, métodos, estrategias y técnicas. Lima, Perú: Universidad Inca Garcilaso de la Vega; 2014.

29. Braun V, Clarke V. Using thematic analysis in psychology. Qual Res Psychol [revista en la Internet]. 2006; 3(2): 77-101. Disponible en: https://www.tandfonline.com/doi/abs/10.1191/14 78088706QP0630A

30. Montero, M. Hacer para transformar. El método de la psicología comunitaria. Primera Edición. 
Buenos Aires, Argentina: Editorial Paidós SAICF; 2006.

31. Organización Mundial de la Salud. Cerrando la brecha: la política de la acción sobre los determinantes sociales de la salud. Conferencia Mundial sobre los Determinantes Sociales de la Salud. Río de Janeiro, Brasil, 19-21 de octubre de 2011. Río de Janeiro, Brasil: OMS; 2011. Disponible en:

https://www.who.int/sdhconference/discussion_p aper/Discussion-Paper-SP.pdf

32. Velásquez F, González E. ¿Qué ha pasado con la participación en Colombia? Bogotá D.C., Colombia: Fundación Corona; 2003. Disponible en: https://www.academia.edu/download/31126817/s ocial36.pdf

33. Colorado A, Bustamante L, Villegas J, Arias LE, Bonilla $M$, Jaramillo $B$, et al. Ideas, decisiones $y$ acciones para el fortalecimiento de la participación comunitaria en salud. Curso-taller para el fortalecimiento del liderazgo y la participación. Primera Edición. Medellín, Colombia: Universidad de Antioquia; $2017 . \quad$ Disponible: https://revistas.udea.edu.co/index.php/fnsp/article /view/328531

34. Colombia. Ministerio de Salud y Protección Social. Resolución 2063 de 2017 por la cual se adopta la política de Participación Social en Salud. Diario Oficial, 50263 (Jun. 9 2017). Disponible en: https://www.asivamosensalud.org/politicaspublicas/normatividad-resoluciones/promocionsocial/resolucion-2063-de-2017-politicade\#: :text=Mediante\%20la\%20Resoluci\%C3\%B3n\% 202063\%20de,sistema\%20de\%20salud\%20en\%20c onjunto.

35. Suárez-Álvarez O, Martínez-Álvarez A, GarcíaBusto B, Palacio-Martín S. Claves para el éxito de la participación comunitaria: diálogos sobre participación en el ámbito local. Informe SESPAS 2018. Gac Sanit. 2018; 32(S1): 48-51. Doi: https://doi.org/10.1016/j.gaceta.2018.06.003

36. Gil-Quevedo W, Agurto-Távara E, EspinozaPortilla E. Ciudadanos informados y empoderados: claves para el pleno ejercicio de los derechos en salud. Rev Peru Med Exp Salud Pública. 2017; 34(2): 311-5.

Doi:

https://doi.org/10.17843/rpmesp.2017.342.2747

37. Mossos-Jiménez MA, Mora-Lemus G. Movimientos sociales subalternos: análisis crítico del discurso del Movimiento Nacional por la Salud y la Seguridad Social en Bogotá. Revista Controversia [revista en la Internet]. 2015; (204): 47-75. Disponible en: https://revistacontroversia.com/index.php?journal $=$ controversia \&page=article\&op=view $\&$ path $\% 5 \mathrm{~B} \% 5$ $\mathrm{D}=184$ 